\title{
A Synthetic Neural Cell Adhesion Molecule Mimetic Peptide Promotes Synaptogenesis, Enhances Presynaptic Function, and Facilitates Memory Consolidation
}

\author{
Karine Cambon, ${ }^{1}$ Stine M. Hansen, ${ }^{2}$ Cesar Venero, ${ }^{1}$ A. Isabel Herrero, ${ }^{1}$ Galina Skibo, ${ }^{2}$ Vladimir Berezin, ${ }^{2}$ \\ Elisabeth Bock, ${ }^{2}$ and Carmen Sandi ${ }^{1,3}$ \\ ${ }^{1}$ Psychobiology Department, Universidad Nacional de Educacion a Distancia, Ciudad Universitaria Sin Numero, 28040 Madrid, Spain, ${ }^{2}$ Protein Laboratory, \\ Institute of Molecular Pathology, School of Medicine, University of Copenhagen, Panum Institute 6.2, DK-2200 Copenhagen N, Denmark, and ${ }^{3}$ Brain and \\ Mind Institute, Ecole Polytechnique Fédérale de Lausanne, CH-1015 Lausanne, Switzerland
}

The neural cell adhesion molecule (NCAM) plays a critical role in development and plasticity of the nervous system and is involved in the mechanisms of learning and memory. Here, we show that intracerebroventricular administration of the FG loop (FGL), a synthetic 15 amino acid peptide corresponding to the binding site of NCAM for the fibroblast growth factor receptor 1 (FGFR1), immediately after training rats in fear conditioning or water maze learning, induced a long-lasting improvement of memory. In primary cultures of hippocampal neurons, FGL enhanced the presynaptic function through activation of FGFR1 and promoted synapse formation. These results provide the first evidence for a memory-facilitating effect resulting from a treatment that mimics NCAM function. They suggest that increased efficacy of synaptic transmission and formation of new synapses probably mediate the cognition-enhancing properties displayed by the peptide.

Key words: NCAM; mimetic peptide; synaptogenesis; synaptic plasticity; spatial learning; fear conditioning; rat

\section{Introduction}

Memory formation is believed to be encoded by changes in the pattern of neural circuits, involving long-lasting, activitydependent changes in the efficacy of synaptic communication (Malenka and Nicoll, 1999; Martin and Morris, 2002). Intensive research over the past two decades has allowed considerable progress in the understanding of the cellular and molecular mechanisms underlying enduring synaptic modifications and memory storage (Elgersma and Silva, 1999; Abel and Lattal, 2001). In addition to events directly involved in cell-signaling mechanisms, a number of other molecular processes are known to play key roles in neural remodeling.

The neural cell adhesion molecule (NCAM), a member of the Ig superfamily expressed on the surface of most neural cells, is involved in cell-cell interactions during brain development, synaptic plasticity, and regeneration (Schachner, 1997; Rønn et al., 2000a). The role of NCAM in cognitive processes is demonstrated by studies in which interference with NCAM function through

Received Dec. 1, 2003; revised March 7, 2004; accepted March 7, 2004.

This work was supported by grants from the Lundbeck Foundation, The Danish Cancer Society, the International Association for the Promotion of Cooperation with Scientists from the Independent States of the Former Soviet Union Project 011-0064, the Spanish Ministry of Science and Technology (MCYT, PM99-027), and the European Union Program (QLK6-computed tomography-1999-02187). C.V. was the recipient of a postdoctoral fellowship from Comunidad de Madrid (Spain). We thank Darya Kiryushko for technical assistance with the presynaptic function and Jason Edamoore for proofreading this manuscript.

Correspondence should be addressed to Carmen Sandi, Laboratory of Behavioral Genetics, Brain and Mind Institute, Swiss Federal Institute of Technology, CH-1015 Lausanne, Switzerland. E-mail: carmen.sandi@epfl.ch.

K. Cambon's present address: Biocortech, Institut Pasteur, 28 rue du Dr Roux, 75015 Paris, France.

G. Skibo's present address: Department of Cytology, Bogomoletz Institute of Physiology, 01024 Kiev, Ukraine.

D0I:10.1523/JNEUROSCI.0436-04.2004

Copyright $\odot 2004$ Society for Neuroscience $\quad$ 0270-6474/04/244197-08\$15.00/0 the administration of antibodies or gene inactivation has resulted in impaired long-term potentiation (LTP) (Luthi et al., 1994; Rønn et al., 1995) and learning and memory deficits (Doyle et al., 1992; Cremer et al., 1994; Sandi et al., 1995). The converse situation, however, i.e., facilitation of memory formation by promoting NCAM function, has not been proven. This is attributable to a large extent to the lack of small synthetic agonists mimicking NCAM interactions, either homophilic (binding with other NCAM molecules on neighboring cells) or heterophilic (binding with NCAM ligands-counter-receptors).

By means of combinatorial chemistry and nuclear magnetic resonance (MR) spectroscopy, synthetic NCAM peptide mimetics have recently become available (Rønn et al., 1999, 2002; Soroka et al., 2002; Kiselyov et al., 2003). The extracellular part of NCAM consists of five Ig (Ig) and two fibronectin type III (F3) modules. After homophilic binding, NCAM promotes neurite outgrowth through mechanisms involving the interaction with the fibroblast growth factor receptor (FGFR) (Saffell et al., 1994; Niethammer et al., 2002; Kiselyov et al., 2003) and the activation of a series of intracellular signaling cascades (Kolkova et al., 2000). The recently identified FG loop (FGL) peptide, a 15 amino acid sequence in the second F3 module of NCAM that represents a part of the binding site of NCAM to the FGFR1, has been shown to bind to and activate FGFR1 and to stimulate neurite outgrowth (Kiselyov et al., 2003). We report here that administration of the NCAM mimetic FGL peptide increases memory strength in rats and enhances presynaptic function in primary hippocampal neurons.

\section{Materials and Methods}

Subjects. Male Wistar rats (Complutense School of Medicine, Madrid, Spain) were received when they were between 6 and 7 weeks of age 
(weight, 160-170 gm). They were housed in groups of three per cage, under light (12 hr light/dark cycle; light on at 7 A.M.)- and temperature (22 \pm $2^{\circ} \mathrm{C}$ )-controlled conditions. Food and water were available ad libitum. Animal care procedures were conducted in accordance with the guidelines set by the European Community Council Directives (86/609/EEC).

Surgery. Approximately 2 weeks after arrival, rats were prepared for intracerebroventricular cannulation. They were anesthetized intraperitoneally with 2,2,2-tribromomethanol (Aldrich, Milwaukee, WI; 250 $\mathrm{mg} / \mathrm{kg}$ ). A 22 gauge Teflon guide cannula (Plastic One, Roanoke, VA) fitted with a removable dummy cannula was stereotactically implanted into the left lateral cerebral ventricle at a position of $1.3 \mathrm{~mm}$ posterior, 1.6 $\mathrm{mm}$ lateral, and $3.5 \mathrm{~mm}$ deep and fixed to two screws in the skull using dental cement (Duralay 2244, Reliance). At least $5 \mathrm{~d}$ were allowed for recovery from surgery before any behavioral test. Once operated, animals were housed individually, and their body weight was monitored.

Preparation of peptides. The FGL pentadecapeptide (EVYVVAENQQGKSKA; Swiss Prot p13596) and the control peptide, FGLala, in which two glutamine residues were substituted with alanines, were synthesized as dendrimers composed of four monomers coupled to a lysine backbone by Loke A/S (Aarhus, Denmark).

Intracerebroventricular injection procedure. The FGL pentadecapeptide and the control peptide FGLala were prepared in an artificial CSF vehicle solution. A flexible swivel attached to the rat allowed the peptide to be administered just after both types of training (fear conditioning and water maze learning) while the animal was conscious and freely moving in a cage. The dummy cannula was removed and replaced with an infusion cannula (Plastic One) attached to a $2.5 \mathrm{ml}$ Hamilton syringe via polyurethane tubing (fluorinated ethylene propylene, $0.12 \mathrm{~mm}$ diameter CMA, Microdialysis AB, Stockholm, Sweden). A microinjection pump (Harvard Apparatus, Cambridge, MA) controlled the delivery of $5 \mu$ l of solution through the cannula, at a rate of $1.61 \mu \mathrm{l} / \mathrm{min}$. The cannula was left in place for an additional 3 min to allow diffusion of the peptide away from the cannula tip before replacement with the dummy cannula. All rats were habituated to this injection procedure (i.e., no solution was injected) for $2 \mathrm{~min}$ daily for $2 \mathrm{~d}$ preceding the proper injection on the day of the experiment.

Fear conditioning. Training and testing took place in a rodent observation cage $(30 \times 37 \times 25 \mathrm{~cm})$ that was placed in a sound-attenuating chamber. The side walls of the observation cage were constructed of stainless steel, and the back walls and doors were constructed of clear Plexiglas. The floor consisted of 20 steel rods through which a $0.4 \mathrm{~mA}$ scrambled shock from a shock generator (model LI100-26 Shocker, LETICA I.C., Madrid, Spain) could be delivered. Each observation cage was cleaned with a $0.1 \%$ acetic acid solution before and after each session. Ventilation fans provided a background noise of $68 \mathrm{~dB}$, and a $20 \mathrm{~W}$ white light-bulb illuminated the chamber.

All animals were handled for 2 min every day for $3 \mathrm{~d}$ preceding the first day of the experiment. They were handled once again for $2 \mathrm{~min}$ on the day before the 1 month test. On the training day of contextual fear conditioning (ConFC), each rat was transported from the colony room to the laboratory (situated in adjacent rooms) and placed in the conditioning chamber. After $3 \mathrm{~min}$, the rats received a single 1 sec shock (unconditioned stimuli) every $\min$ for $2 \mathrm{~min}$. They were removed from the conditioning chambers $30 \mathrm{sec}$ after the final shock presentation. Thus, a conditioning session lasted $\sim 330 \mathrm{sec}$. Animals were then injected with $5 \mu$ l of the FGL peptide $(5 \mu \mathrm{g})$, the control peptide FGLala $(5 \mu \mathrm{g})$, or the vehicle solution in an adjacent room, before returning to their home cage. Testing for ConFC was performed on the following day, as well as 1 week and 1 month after the training day. At testing, rats were placed again in the chamber used for conditioning, but in the absence of shock, for an 8 min context test.

In the cued fear conditioning (CueFC), training consisted of exposure of the rats to a conditioning chamber $(160 \mathrm{sec})$, followed by a tone $(85 \mathrm{~dB}$ sound at $1000 \mathrm{~Hz}$ ) that was presented for $20 \mathrm{sec}$ before the electric footshock ( $1 \mathrm{sec}, 0.4 \mathrm{~mA}$, constant current). Each rat received three $1 \mathrm{sec}$ shocks with an intertrial interval of $60 \mathrm{sec}$. The rats were removed from the conditioning chamber $30 \mathrm{sec}$ after the final shock and returned to their home cages. Thus, a conditioning session lasted $\sim 5.5 \mathrm{~min}$. One day after conditioning ( $24 \mathrm{hr}$ test), the rats were placed in a novel context ( 3 $\mathrm{min}$ ) and re-exposed to the tone ( $3 \mathrm{~min}$ ). Subsequently, 1 week and $28 \mathrm{~d}$ later ( 1 week test and $28 \mathrm{~d}$ test, respectively), the rats were placed in a novel context, different from the one previously used in the $24 \mathrm{hr}$ test. After a $3 \mathrm{~min}$ baseline period, the training tone was played for $3 \mathrm{~min}$. In addition, 1 month after training, they were re-exposed to the context in which they originally received the tone-footshock pairings, but in the absence of the conditioning tone.

Using a time-sampling procedure, every $2 \mathrm{sec}$, each rat was scored blindly as either freezing or active. Freezing was defined as behavioral immobility except for movement needed for respiration. At training, behavioral scores were performed for the 3 min period before shock and for the $2.5 \mathrm{~min}$ period starting immediately after presentation of the first shock ("after shock" period). Scores for each of these periods were analyzed separately. At testing, freezing was scored during the 8 min reexposure to the training context. All experiments were conducted between 9:00 A.M. and 12:00 P.M. to avoid the influence of circadian hormonal fluctuations.

Spatial learning in the Morris water maze. The water maze was a black circular pool ( $2 \mathrm{~m}$ diameter, $45 \mathrm{~cm}$ high) filled with water $(30 \mathrm{~cm}$ depth, at $25^{\circ} \mathrm{C}$ ). The pool was divided into four quadrants of equal size. An invisible escape platform ( $11 \mathrm{~cm}$ diameter) was placed in the middle of one of the quadrants ( $1.5 \mathrm{~cm}$ below the water surface) equidistant from the sidewalls and the middle of the pool. The testing room contained numerous extra maze cues. The behavior of the animal (latency to reach the platform, distance, and swim speed) was monitored by a video camera, mounted on the ceiling above the center of the pool, and a computerized tracking system (Ethovision 1.90, Noldus IT, Wageningen, The Netherlands).

Rats were handled daily for 2 min for $5 \mathrm{~d}$ preceding the training. At training, rats were given a block of three consecutive trials every day for $2 \mathrm{~d}$. The starting position differed for each trial but was identical for all animals, with rats placed in the water facing the wall of the pool. The platform location remained constant throughout training. Each trial lasted $120 \mathrm{sec}$ or until the animal located the platform. Animals that did not find the platform were guided to it and given a latency score of 120 sec. All animals were left on the platform for $30 \mathrm{sec}$ and then taken away from the pool for a $30 \mathrm{sec}$ rest period before starting the next trial. After the last trial, the rats were returned to their home cage in a heated room to dry. They received a $5 \mu$ injection immediately after each training day, with the FGL peptide $(5 \mu \mathrm{g})$, the control peptide FGLala $(5 \mu \mathrm{g})$, or the vehicle solution.

Twenty four hours and 1 week after training, animals were given one trial followed by a $60 \mathrm{sec}$ probe trial during which the platform was removed from the pool. The probe trial started from a position in a quadrant adjacent to the quadrant that originally contained the platform. At the end of the trial, the platform was raised. The rat was guided to it and allowed to stay there for $30 \mathrm{sec}$. The percentage of time spent in each quadrant was recorded.

Two weeks after training, rats were given one trial followed by a block of three consecutive trials to learn a new location of the platform ("reversal learning"). The starting position differed for each trial and from the training day but was identical for all rats. The procedure for each trial was identical to those used for training.

Open field. The emotional behavior and locomotor activity of the rats submitted to the water maze test were assessed in the open field in the afternoon of the $24 \mathrm{hr}$ test. The structure of the open field consisted of a circular arena ( $1.40 \mathrm{~m}$ diameter, $32 \mathrm{~cm}$ high). For analysis, the floor was divided into two virtual concentric parts, with an inner zone in the middle of the arena ( $0.7 \mathrm{~m}$ diameter) and an outer zone as the remaining area along the sidewalls. At the start of testing, the animals were placed in the center of the arena. The locomotor activity was monitored for $5 \mathrm{~min}$ by a video camera, mounted on the ceiling above the center of the arena, and a computerized tracking system (Ethovision 1.50, Noldus IT) recorded total locomotion, speed, percentage of time spent in each zone, number of crossings between inner and outer zones, and freezing time. Defecations were also counted by the experimenter. The floor of the arena was washed with $0.1 \%$ acetic acid solution to remove odors left by previous subjects.

Control for cannula placement. At the end of all experiments, rats were anesthetized with an overdose of 2,2,2-tribromomethanol (Aldrich). A cresyl violet solution was administered intracerebroventricularly in the same way as the FGL peptide and the control solutions. After decapitation, the brains were removed and examined for the presence of cresyl 
violet in the third ventricle. Only the rats with correct cannula implantation were included in the analyses.

Primary cultures of hippocampal neurons. Hippocampal neurons were prepared from embryonic day 19 rat embryos as described previously (Maar et al., 1997). Neurons were plated at a density of 50,000 cells $/ \mathrm{cm}^{2}$ in eight-well LabTek tissue culture chambers (Nunc, Roskilde, Denmark) coated with poly-L-lysine. Cultures were grown at $37^{\circ} \mathrm{C}$ in a humidified atmosphere with $5 \% \mathrm{CO}_{2}$ in neurobasal medium containing $20 \mathrm{mM}$ HEPES, $100 \mathrm{U} / \mathrm{ml}$ penicillin, $100 \mu \mathrm{g} / \mathrm{ml}$ streptomycin (all from Invitrogen, Copenhagen, Denmark), and 0.4\% w/v bovine serum albumin (Sigma, Copenhagen, Denmark), supplemented with B27 (Invitrogen). The cultures were grown for 2 weeks before the addition of peptides. Half of the medium was replaced with fresh medium $3 \mathrm{~d}$ after plating and again $6 \mathrm{~d}$ later.

Analysis of presynaptic function with FM1-43. At 14-16 d in vitro, hippocampal cultures were labeled with the fluorescent styryl membrane probe FM1-43 (Molecular Probes, Eugene, OR) as described previously (Ryan et al., 1993; Kiryushko et al., 2003). In brief, synapses were loaded with FM1-43 by adding a $90 \mathrm{~mm} \mathrm{KCl}$ solution containing $5 \mu \mathrm{M}$ FM1-43 for $60 \mathrm{sec}$. To remove surface-bound FM1-43, the cultures were subsequently washed three times for 4 min in HBSS (Sigma) with the $\mathrm{Ca}^{2+}$ concentration adjusted to $1.8 \mathrm{~mm}$ and $\mathrm{pH}$ adjusted to 7.3.

Immunofluorescence. Immunostaining for GAP43 and synaptophysin was done as described previously (Kiryushko et al., 2003). In brief, fixed cell cultures were incubated with rabbit anti-rat-GAP43 polyclonal antibodies [(1:1000, prepared as described previously (Mosevitsky et al., 1997)] and anti-synaptophysin mouse monoclonal antibody (IgG; diluted 1:500; Sigma). Bound antibodies were detected with fluoresceinconjugated goat anti-rabbit IgG (1:200; Molecular Probes) or Texas Redconjugated goat anti-mouse IgG (1:100; Molecular Probes), respectively. Mounted slides were scanned with a Radiance 2000 scanning system (Bio-Rad, Hertfordshire, UK) and a Nikon eclipse TE200 confocal microscope equipped with an oil immersion, $60 \times, 1.4$ numerical aperture objective (Nikon, Tokyo, Japan).

Image analysis. To evaluate the rate of FM1-43 release, cultures labeled with FM1-43 were treated for $100 \mathrm{sec}$ with $90 \mathrm{~mm} \mathrm{KCl}$ and studied by confocal microscopy using time-lapse image acquisition. The excitation wavelength was $488 \mathrm{~nm}$. Time-lapse series recording and quantification of FM1-43 destaining were performed using the Lasersharp 2000 software package (Bio-Rad). After FM1-43 loading, a field of interest (FOI) containing at least 15 punctate-like areas was chosen, and five consecutive scans of the synapses in the FOI were obtained, with one image taken every $5 \mathrm{sec}$. After $25 \mathrm{sec}$, the $90 \mathrm{~mm} \mathrm{KCl}$ solution was added, and the FOI was scanned every $5 \mathrm{sec}$ for $100 \mathrm{sec}$ to measure the dye release. To evaluate the rate of FM1-43 unloading, the experimentally obtained data were approximated to a single exponential function, $\exp (-k t)$, where $k$ is the rate of FM1-43 unloading and $t$ is the time elapsed from the addition of $90 \mathrm{~mm} \mathrm{KCl}$.

To evaluate synapse formation, cultures of hippocampal neurons grown for 2 weeks were double-stained for GAP43 (which stains neuronal cell bodies and neurites) and synaptophysin (which is present in presynaptic areas). The total length of GAP43-stained neurites (micrometers; referred to as $\mathrm{Nn}$ ) in each video-recorded image was quantified using a stereological approach as described previously (Rønn et al., $2000 \mathrm{~b}$ ). The number of synaptophysin-positive spots (in red; referred to as $\mathrm{Ns}$ ) coinciding with GAP43-stained neurites (in green) in each image was counted using a computer mouse. This analysis was performed in a "blind" manner by three independent experimenters working on numbered images. A ratio $N s / N n \times 100$ (number of synaptophysin-positive spots per $100 \mu \mathrm{m}$ neurite length) was calculated as described previously (Kiryushko et al., 2003).

Data analysis. Statistics were performed using the Origin version 6.1. software package (Originlab) and SPSS version 10 (Chicago, IL). The results were expressed as mean \pm SEM and analyzed using unpaired Student's $t$ test, one-way ANOVA, or a repeated measure ANOVA where appropriate. Significance of results was accepted at $p<0.05$.

\section{Results}

FGL enhances contextual fear conditioning

Training rats in the ConFC task under experimental conditions leading to a robust memory (unconditioned stimulus: $1 \mathrm{~mA}$
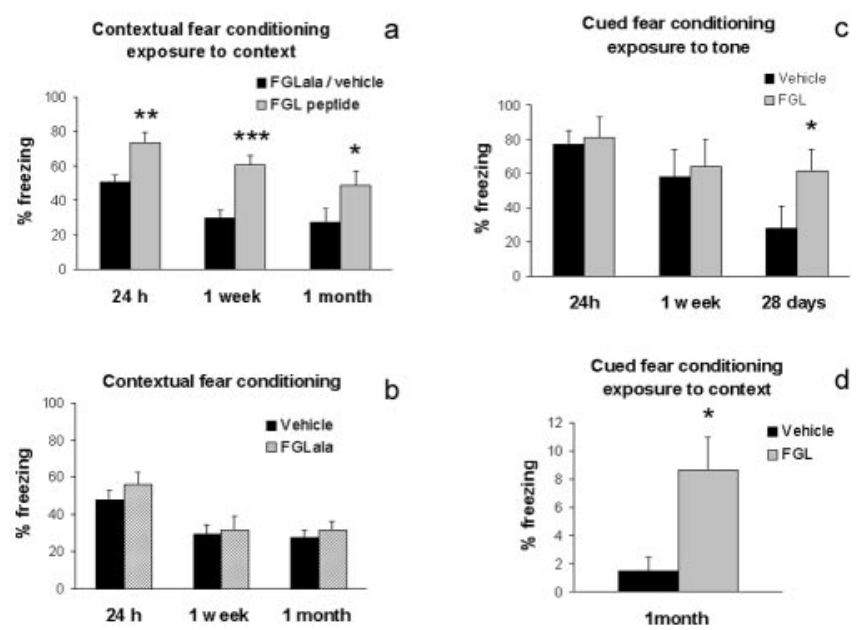

Figure 1. FGL enhances memory for context. $a, b, \ln$ a ConFC test, rats received footshocks in the conditioning chamber and were injected intracerebroventricularly with $5 \mu \mathrm{g}$ of the FGL peptide $(n=11)$, vehicle $(n=18)$, or control peptide FGLala $(n=10)$ immediately after the conditioning session. They were tested in the conditioning chamber $24 \mathrm{hr}, 1$ week, and 1 month later. Data from vehicle and FGLala groups are merged into a single control group $(a)$, because no difference was observed $(b)$ between them. $c, d$, In a CueFC test, rats received pairings of tone and footshocks in the conditioning chamber. They were injected intracerebroventricularly with $5 \mu \mathrm{g}$ of the FGL peptide $(n=6)$ or vehicle $(n=6)$ immediately after the conditioning session. They were tested in a different chamber in the presence of a tone $24 \mathrm{hr}, 1$ week, and $28 \mathrm{~d} \mathrm{later}$ (c) or in the original conditioning chamber in the absence of a tone 1 month later ( $d$ ). Data are mean percentage of time spent freezing $\pm \mathrm{SEM}^{*}{ }^{*} p<0.05$, ${ }^{* *} p<0.01$, and ${ }^{* * *} p<0.005$, when compared with control group.

shock) has previously been shown to induce enhanced hippocampal expression of NCAM $24 \mathrm{hr}$ after training (Merino et al., 2000), in agreement with lesion studies indicating that the hippocampus is critically involved in this task (Phillips and Ledoux, 1992). Because the FGL peptide has been shown to mimic some functions characteristic of NCAM (Kiselyov et al., 2003), we reasoned that with a training procedure that results in only moderate levels of fear conditioning, FGL treatment might strengthen memory. To address this issue, adult male rats were trained in the ConFC task with a $0.4 \mathrm{~mA}$ shock intensity and then immediately injected intracerebroventricularly with $5 \mu \mathrm{g}$ of FGL $(n=11)$, control peptide FGLala $(n=10)$, or vehicle $(n=18)$. At training, and therefore before injections were given, all groups of rats displayed equivalent levels of freezing during the post-shock period $(t=2.08 ; \mathrm{df}=37 ; p=0.16$; data not shown). In contrast, post-training FGL significantly enhanced the retention of the conditioned freezing response for at least 1 month after treatment $\left(F_{(1,37)}=13.36 ; p<0.001\right)$, as indicated by elevated freezing values of FGL-injected rats when re-exposed to the conditioning context at $24 \mathrm{hr}(t=3.01 ; \mathrm{df}=37 ; p<0.005)$, 1 week $(t=$ 3.83 ; $\mathrm{df}=37 ; p<0.0005)$, and 1 month $(t=2.18$; $\mathrm{df}=37 ; p<$ 0.05 ) after training (Fig. 1a). Because data obtained from control peptide- and vehicle-treated rats were equivalent at all testing times $\left(F_{(1,26)}=0.514 ; p=0.48\right)$ (Fig. $\left.1 b\right)$, only a vehicle control group was used in the following experiments, to minimize the number of animals (Fig. $1 c, d$ ). For presentation purposes, Figure $1 a$ shows the merged data from FGLala and vehicle groups.

We then examined whether the same treatment could also affect a hippocampus-independent task using auditory CueFC. Lesion studies indicate that whereas both the contextual and the auditory-cued versions of the fear-conditioning task are dependent on the amygdala, only the contextual variant is dependent on the hippocampus (Phillips and Ledoux, 1992). Rats were in- 
jected intracerebroventricularly with $5 \mu \mathrm{g}$ of FGL $(n=6)$ or vehicle $(n=6)$ immediately after being trained in the auditorycued version of this task with $0.4 \mathrm{~mA}$ shocks. These groups did not differ in their post-shock freezing values at training $(t=$ 1.376; $\mathrm{df}=10$; NS; data not shown). In contrast to the effect exerted on ConFC, post-training FGL injections did not influence subsequent retention of the auditory $\operatorname{CueFC}\left(F_{(1,10)}=0.823\right.$; $\mathrm{NS})$ at $24 \mathrm{hr}(t=0.258 ; \mathrm{df}=10 ; \mathrm{NS})$ and $7 \mathrm{~d}(t=0.263 ; \mathrm{df}=10$; NS) (Fig. 1c); however, when they were tested again $28 \mathrm{~d}$ after training, FGL-injected rats displayed higher freezing responses to the conditioning tone than did control rats $(t=2.802 ; \mathrm{df}=9 ; p<$ 0.02 ; one rat from the vehicle group was removed from the analyses because it exhibited very high levels of freezing before exposure to the tone in this test). Therefore, although FGL treatment did not influence the expression of auditory fear conditioning during the first week after training, it potentiated long-term retrieval at a time when the percentage of freezing of control animals had decayed considerably because of forgetting or extinction processes, or both (i.e., $28 \mathrm{~d}$ after training). This long-term increased freezing of FGL-treated rats could have been caused by a failure in extinction learning to the repeated presentation of the conditioning tone in the absence of shock. Therefore, $2 \mathrm{~d}$ afterward, the same animals were re-exposed to the context in which they had originally received ( 1 month before) the tone-footshock pairings, but this time in the absence of the conditioning tone (Fig. 1d). Interestingly, the percentage of freezing displayed in the conditioning context was markedly higher in FGL-treated than in control rats $(t=$ 2.519; $\mathrm{df}=9 ; p<0.05)$, which suggests that FGL treatment increases the strength of the fear memory developed, making it more persistent. Additional tests performed 2 months after training indicated that freezing values of FGL-treated rats no longer differed from controls when the rats were once more confronted with the auditorycued and contextual tests (data not shown).

\section{FGL improves spatial learning}

To explore whether the performance of rats in another hippocampus-dependent learning task, the water maze (Morris et al., 1982), could also benefit from FGL treatment, we evaluated the effect of post-training administration of the synthetic peptide on retrieval and subsequent relearning of this task. Thus, in the first session, we trained rats for three consecutive trials to find a submerged platform in the water maze and immediately afterward injected them intracerebroventricularly with $5 \mu \mathrm{g}$ of FGL $(n=12)$, control peptide FGLala $(n=13)$, or vehicle $(n=23)$. As can be seen in Figure 2, $a$ and $b$, all groups were equivalent in their latencies to find the platform during the first training session (i.e., before any drug treatment). When tested $24 \mathrm{hr}$ later, however, FGL-injected rats when compared with controls exhibited decreased latencies to find the platform in the first trial of that session (i.e., trial 4 of the training protocol) $(t=2.269$; $\mathrm{df}=46$; $p<0.05$ ), indicating a better memory for the location of the platform in FGL peptide-treated animals. Nevertheless, their performance normalized to that of controls through the following two trials of the session (NS), and immediately after the last trial (trial 6), all rats were subjected to the same regimen of injections as on the previous day.

After these 2 training days, the effects of post-training FGL injections were firstly evaluated with a single relearning trial 24 hr, 1 week, and 2 weeks later. The platform was located in the same place as before (Fig. 2a). A repeated measure ANOVA revealed a significant effect of treatment $\left(F_{(1,20)}=19.881 ; p<\right.$ $0.0005)$. FGL-injected rats found the platform twice as fast as the control group when tested at the $24 \mathrm{hr}(t=1.952$; $\mathrm{df}=45 ; p<$
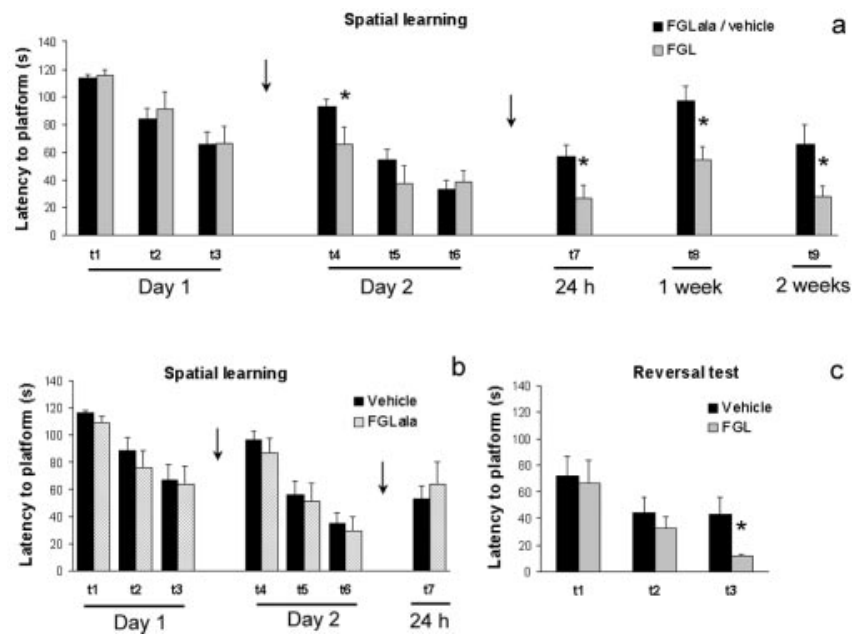

Figure 2. FGL improves spatial learning. $a, b$, Mean escape latencies (sec) to find the platform \pm SEM are represented for the vehicle $(n=23)$, control peptide $(n=13)$, and FGL peptide ( $n=12$ ) groups over the 2 training days ( 3 trials each) and all of the testing trials performed $1 \mathrm{~d}, 1$ week, and 2 weeks after training. A $5 \mu \mathrm{g}$ injection of either vehicle, control peptide FGLala, or FGL peptide was given immediately after each training day (arrow). Data from vehicle and FGLala groups are merged into a single control group ( $a$ ), because no difference was observed $(b)$ between them. $c$, Two weeks after training, animals were submitted to a reversal learning task ( 3 trials) in which the platform was placed in a new quadrant of the pool. ${ }^{*} p<0.05$, when compared with control group.

$0.05), 1$ week $(t=2.921 ; \mathrm{df}=36.08 ; p<0.01)$, and 2 weeks $(t=$ $2.331 ; \mathrm{df}=15,54 ; p<0.05)$ retention times. Probe tests after each single relearning trial at $24 \mathrm{hr}$ and 1 week showed no difference between the groups $(t=0.650 ; \mathrm{df}=22 ; p=0.52$; and $t=1.091$; $\mathrm{df}=45 ; p=0.28$, respectively; data not shown). Again, because data obtained from control peptide- and vehicle-treated rats were equivalent during both days of training (day $1: F_{(1,34)}=0.715$, $p=0.4$; day 2: $\left.F_{(1,34)}=0.331, p=0.57\right)$ and for the $24 \mathrm{hr}$ test $(t=$ $0.598 ; \mathrm{df}=33 ; p=0.55$ ) (Fig. $2 b$ ), only the vehicle control group was put through the remaining tests ( 1 week, 2 weeks, and 1 month) for comparison with the FGL group. For presentation purposes, Figure $2 a$ shows the merged data from FGLala and vehicle groups.

Subsequently, we evaluated whether FGL treatment also had an effect on behavioral flexibility (Fig. 2c). Immediately after animals were tested for their memory of the location of the platform in trial 9 (i.e., 2 weeks after training), they were submitted to a reversal learning task, in which the platform was placed in a new quadrant of the pool. The FGL group was quicker than controls to acquire the new spatial response, finding the platform approximately four times faster than the controls in their third reversal trial $(t=2.53$; df $=11.18 ; p<0.05)$. Finally, we also checked whether differences in swimming ability could account for the observed variations in escape latencies; however, no difference in swimming speed for each individual trial of the whole experiment was observed between the different groups (NS; data not shown).

\section{FGL does not alter emotional behavior and locomotion}

Given that treatments modulating emotional or locomotor responsiveness could affect the performance in the behavioral tasks used in this study, we assessed whether injections of the FGL peptide could influence the behavior in an open field task. Thus, rats received intracerebroventricular injections on 2 consecutive days of either the FGF peptide ( $5 \mu \mathrm{g} ; n=12)$ or vehicle $(n=12)$. As shown in Table 1, no difference was observed between the FGL and control groups for any of the parameters evaluated (locomotion, 
Table 1. Intracerebroventricular injections of FGL had no effect on emotional behavior and locomotor activity in the open field

\begin{tabular}{|c|c|c|c|c|c|c|c|c|c|}
\hline & $n$ & Locomotion (cm) & Speed $(\mathrm{cm} / \mathrm{s})$ & \% Freezing & $\%$ Time in inner zone & $\%$ Time in outer zone & $\begin{array}{l}\text { Number of } \\
\text { zone crossings }\end{array}$ & $\begin{array}{l}\text { Number of } \\
\text { defecations }\end{array}$ & $\begin{array}{l}\% \text { Change in weight after } \\
\text { two injections }\end{array}$ \\
\hline Vehicle & 12 & $2852 \pm 161$ & $9.53 \pm 0.54$ & $1.65 \pm 0.52$ & $4.28 \pm 0.80$ & $95.72 \pm 0.80$ & $4.50 \pm 0.78$ & $3.36 \pm 0.66$ & $1.67 \pm 0.30$ \\
\hline FGL & 12 & $3111 \pm 224$ & $10.39 \pm 0.75$ & $0.92 \pm 0.33$ & $3.74 \pm 0.60$ & $96.24 \pm 0.60$ & $5.83 \pm 0.72$ & $3.50 \pm 0.77$ & $1.35 \pm 0.35$ \\
\hline
\end{tabular}

Data are mean \pm SEM

a
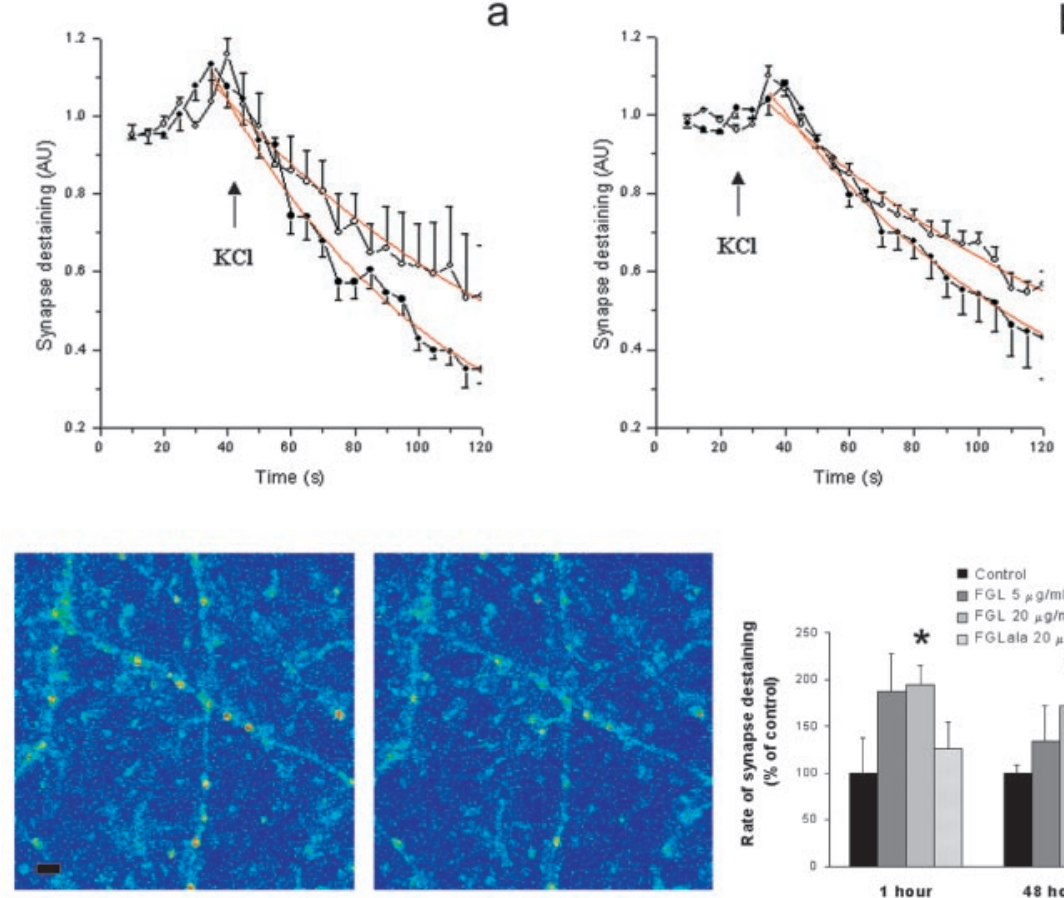

C

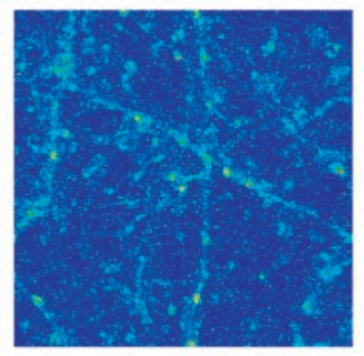

d

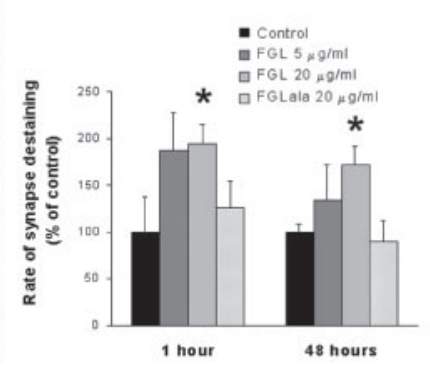

Figure 3. FGL enhances presynaptic function. $a, b$, Effect of FGL on synapse destaining in hippocampal cultures loaded with FM1-43. After FM1-43 loading, an FOI was chosen for scanning and after $20 \mathrm{sec}$, the $90 \mathrm{~mm} \mathrm{KCl}$ solution was added and the F0I was further scanned every $5 \mathrm{sec}$ for $100 \mathrm{sec}$ to measure the dye release. Fluorescence intensity of FM1-43 staining in 15-20 synapses was measured and normalized to the intensity at the start of scanning. The mean intensity for each time point was calculated and approximated to a single exponential function ( $a, b$, red curve) to determine the rate of destaining. Synapses in the hippocampal culture treated with $20 \mu \mathrm{g} / \mathrm{ml} \mathrm{FGL} \mathrm{for} 1 \mathrm{hr}(a)$ and $48 \mathrm{hr}(b)$ were destained faster (filled circles) than synapses in the control cultures (open circles). Results from four to six independent experiments are expressed as a percentage \pm SEM, with untreated controls set at $100 \%$. c, d, Staining and destaining of synapses. Confocal images of hippocampal cultures showing synapses loaded with FM1-43 (c) and synapse destaining $100 \mathrm{sec}$ after start of stimulation with $90 \mathrm{~mm} \mathrm{KCl}$ (d). Scale bar, $8 \mu \mathrm{m}$. $e$, Effect of FGL on the rate ( $k$ ) of FM1-43 unloading. The experimental data obtained as described in $a$ and $b$ were approximated to a single exponential function, $\exp (-k t)$, where $k$ is the rate of FM1- 43 unloading and $t$ is the time elapsed from the addition of $90 \mathrm{~mm} \mathrm{KCl}$. Results from five to eight independent experiments are expressed as a percentage $\pm \mathrm{SEM}$, with untreated controls set at $100 \%$. ${ }^{*} p<0.05$ when compared with untreated control at the same time point.

speed, percentage of freezing, percentage of time spent in either the inner or the outer zone, zone crossings, and defecations). In addition, the two peptide injections did not alter weight gain, and we observed no signs of illness or overt behavioral changes in any of the groups in their home cages throughout the experiment.

\section{FGL enhances presynaptic function}

Activity-dependent changes in synaptic efficacy are believed to be important for learning and memory (Zakharenko et al., 2001). To investigate whether FGL can affect synaptic plasticity in vitro, we used an approach directly allowing the imaging of presynaptic function. Primary cultures of hippocampal neurons were treated for a short time with the fluorescent dye FM1-43 under stimulation with a high concentration of $\mathrm{KCl}$. This resulted in a loading of synaptic vesicles in presynaptic terminals by endocytosis after the presynaptic stimulation and transmitter release (Ryan et al., e

1993). Subsequent addition of $90 \mathrm{~mm} \mathrm{KCl}$ to the medium initiated the release of transmitter from the loaded vesicles, and the rate of this process was measured as the rate of FM1-43 unloading (synapse destaining). Representative micrographs of hippocampal cultures containing synapses loaded with FM1-43 before and 100 sec after destaining was initiated are shown in Figure 3, $c$ and $d$, respectively. Treatment of hippocampal cultures for $1 \mathrm{hr}$ with $20 \mu \mathrm{g} / \mathrm{ml} \mathrm{FGL} \mathrm{resulted} \mathrm{in} \mathrm{a} \mathrm{statisti-}$ cally significant increase of the rate of FM1-43 unloading (Fig. 3a), and this effect was dose dependent, a concentration of $20 \mu \mathrm{g} / \mathrm{ml}$ FGL being more efficient than $5 \mu \mathrm{g} / \mathrm{ml} \mathrm{FGL} \mathrm{(Fig.} \mathrm{3e).} \mathrm{FGL} \mathrm{treatment} \mathrm{of}$ hippocampal neurons for $48 \mathrm{hr}$ also resulted in a faster dose-dependent synapse destaining than that observed in control cultures (Fig. 3b), indicating that FGL causes both a short-term facilitation of transmitter release $(1 \mathrm{hr})$ and a long-term $(48 \mathrm{hr})$ increase of synaptic efficacy (Fig. $3 e$ ). Treatment of hippocampal cultures with the control peptide, FGLala, had no effect on FM1-43 unloading (Fig. 3e).

\section{FGL-enhanced transmitter release} requires activation of the FGF receptor The FGL peptide is a 15 amino acid sequence in the second fibronectin type III module of NCAM, which has recently been shown to represent a part of the binding site for FGFR1. We therefore tested whether the FGL-enhanced rate of FM1-43 unloading was dependent on FGF receptor activation. Hippocampal cultures were treated with $20 \mu \mathrm{g} / \mathrm{ml} \mathrm{FGL}$ for $48 \mathrm{hr}$ in the presence of a specific FGFR1 inhibitor, SU5402 $(10 \mu \mathrm{g} / \mathrm{ml})$, before starting FM1-43 unloading with a high $\mathrm{KCl}$ concentration. In the presence of SU5402, the FGL-induced change in transmitter release was not observed, indicating that FGL modulates presynaptic function via activation of the FGF receptor (Fig. 4).

\section{FGL promotes synapse formation}

Because the FGL peptide modulates synaptic strength, it seemed important to elucidate whether treatment of hippocampal neurons with FGL could also modulate the number of synapses. The number of synapses in cultured neurons correlates with focal accumulations of synaptic vesicle proteins such as synaptophysin. Therefore, counting of synaptophysin-positive spots provides an estimate of the number of presynaptic terminals (Hiscock et al., 2000). After $14 \mathrm{~d}$ in vitro, hippocampal cultures were treated with $20 \mu \mathrm{g} / \mathrm{ml} \mathrm{FGL}$ for 2 or $4 \mathrm{~d}$, after which cultures were 


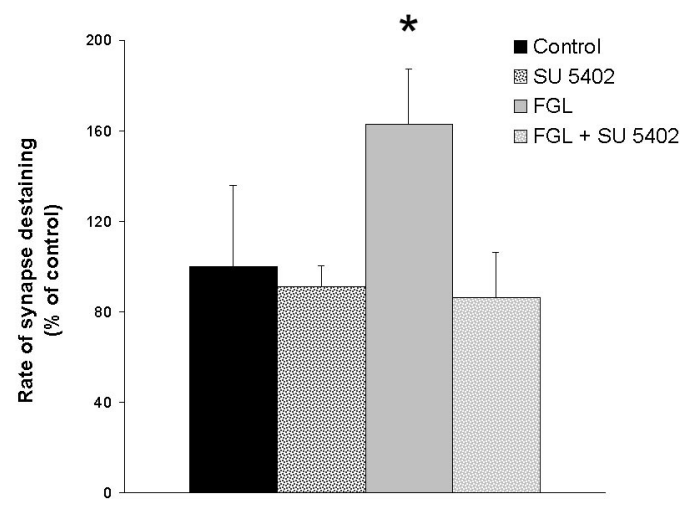

Figure 4. The FGL-enhanced presynaptic function is abrogated by the FGFR inhibitor SU5402. Hippocampal cultures were treated with $20 \mu \mathrm{g} / \mathrm{ml} \mathrm{FGL} \mathrm{for} 48 \mathrm{hr}$ in the presence or absence of $10 \mu \mathrm{g} / \mathrm{ml}$ SU5402. Results from five to eight independent experiments are expressed as a percentage \pm SEM, with untreated controls set at $100 \% .{ }^{*} p<0.05$ when FGL plus SU5402-treated cells were compared with FGL-treated cells.

fixed and double-immunostained for GAP-43 and synaptophy$\sin$ (Fig. $5 b, c)$. We evaluated the number of synaptophysinpositive spots per $100 \mu \mathrm{m}$ neurite length in control and FGLtreated cultures. Treatment of hippocampal cultures with FGL for 2 and $4 \mathrm{~d}$ resulted in a statistically significant increase in density of synaptophysin-positive spots to 122 and $128 \%$ of the control, respectively, indicating that FGL promotes synapse formation (Fig. 5a).

\section{Discussion}

Nuclear MR analyses have revealed a region in the second F3 module of NCAM that binds directly to FGFR1. The synthetic FGL peptide that corresponds to residues $\mathrm{E}^{681}-\mathrm{A}^{695}$ of NCAM, binds to, phosphorylates, and activates FGFR1. In this respect, FGL mimics the heterophilic NCAM interaction with FGFR. Moreover, it reproduces one of the characteristic functions of NCAM, i.e., stimulation of neurite outgrowth (Kiselyov et al., 2003).

We show here that FGL effectively facilitates memory processes, supporting the view that NCAM-FGFR1 interactions critically modulate the strength at which learned responses are stored. Although a role for NCAM in memory formation has been suggested by studies in which interference with NCAM function resulted in the impairment of both learning (Mileusnic et al., 1995; Arami et al., 1996; Foley et al., 2000; Cambon et al., 2003) and synaptic plasticity (Luthi et al., 1994; Cremer et al., 1998), indisputable proof that the mimicking of NCAM function results in memory enhancement has been lacking. Our findings provide the first evidence for a functional significance of NCAM-FGFR interactions in vivo. By extending the triggering mechanism to the binding site of NCAM to FGFR1, our current data further support a facilitating role of FGFR1 in long-term memory formation.

The fact that post-training intracerebroventricular injections of FGL induced a long-lasting facilitation of performance in two hippocampus-dependent tasks (ConFC and Morris water maze) highlights the hippocampus as a likely target for the peptide. In both tasks, the facilitation of memory was already evident at the $24 \mathrm{hr}$ post-training retrieval tests and remained potentiated during the subsequent tests performed up to $14 \mathrm{~d}$ (water maze) and $28 \mathrm{~d}$ (ConFC) after training. Although a proactive effect on retrieval cannot be excluded, the pattern of effects favors the view that FGL affected memory consolidation processes occurring in the hippocampus.

The peptide may also act on other brain regions because long-

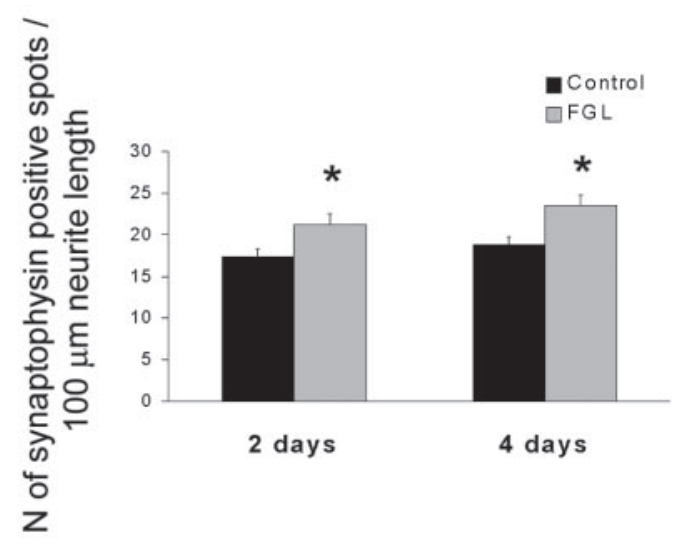

a
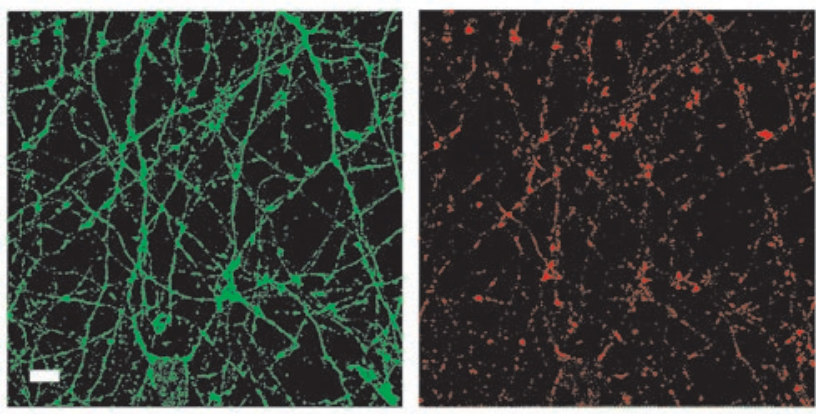

b

C

Figure 5. FGL promotes synaptogenesis. $a$, Effect of FGL on synaptophysin expression in primary hippocampal neurons. At $14 \mathrm{~d}$ in vitro, hippocampal cultures were treated with 20 $\mu \mathrm{g} / \mathrm{ml} \mathrm{FGL} \mathrm{for} 2$ and $4 \mathrm{~d}$ and double-immunostained for GAP43 and synaptophysin. The number of synaptophysin-positive spots per $100 \mu \mathrm{m}$ neurite length was evaluated. Results from a total of nine individual cultures ( 3 independent experiments for each condition) are expressed as means \pm SEM. ${ }^{*} p<0.05$ when compared with untreated control at the same time point. $b, c$, Confocal images of $14 \mathrm{~d}$ in vitro hippocampal cultures immunostained for GAP43 ( $b$, green) and synaptophysin (c, red). Scale bar, $8 \mu \mathrm{m}$.

term decay of memory for the hippocampus-independent auditory CueFC observed in controls $28 \mathrm{~d}$ after training was also prevented by post-training FGL injections. This result might seem surprising because, at shorter retention times, performance only benefited from FGL treatment in animals dealing with hippocampus-dependent tasks; however, the amygdala may also be involved in the potentiation of memory induced by FGL during the consolidation period. Although contextual and auditorycued versions of fear conditioning are frequently referred to as "hippocampus-dependent" and "amygdala-dependent" tasks, respectively, the amygdala is also required for contextual fear conditioning (Kim and Fanselow, 1992; Phillips and Ledoux, 1992). Furthermore, modulatory influences of the amygdala on hippocampal treatments leading to the facilitation of long-term memory for the water maze have also been reported (Roozendaal and McGaugh, 1997). Conversely, evidence indicates that the amygdala does not work alone on the formation of auditory-cued fear-conditioning memory. Other brain areas, including some cortical regions, have also been involved in the formation (Sacchetti et al., 2002) and long-term storage (Quirk et al., 1997) of such cued memories. Nevertheless, the question still remains as to why the effect of FGL in cued conditioning was observed only at the $28 \mathrm{~d}$ post-training retention test but not at earlier times. One possibility is that FGL interfered with extinction learning (Rescorla, 1996). Because our procedures were not designed to evaluate extinction, we cannot determine whether diminished 
performance at $28 \mathrm{~d}$ is the result of forgetting or extinction processes, or both. The fact that FGL-treated rats showed a progressive reduction of their fear response when exposed repeatedly to the conditioning stimulus in the absence of footshock, however, indicates that extinction learning was taking place. A more plausible hypothesis to account for the delayed effect of FGL peptide on cued conditioning is that a stronger memory is formed originally that is more resistant to decay with time. In support of this view is the finding that FGL-treated animals trained in the auditory fear-conditioning task also showed increased memory for the conditioning context 1 month later, when re-exposed for the first time to the context only (in the absence of tone). The strength of a memory includes two independent components (Sandi, 1998): (1) the magnitude at which the behavioral response is expressed at the retention tests, and (2) the duration or length of time for which the memory can be retrieved. A better encoding during consolidation does not need to be reflected exclusively by increased response expression during early retention, particularly when retention levels in controls are high, as is the case in our CueFC experiment (although post-training FGL administration was found to potentiate $24 \mathrm{hr}$ retention for ConFC under training conditions, leading to high conditioning levels in controls; our unpublished observations). Instead, a better encoding could extend the time length at which the information is accessible to retrieval. This type of delayed effect of a treatment on retention has been reported, for example, for a facilitating action of epinephrine injections given to rats after active avoidance training, which was observed at 20 and $45 \mathrm{~d}$ after training but not at earlier testing times: 1 and $11 \mathrm{~d}$ after training (Torras-Garcia et al., 1997).

Post-training FGL injections might facilitate memory storage by affecting the molecular signal cascades elicited at training. Moreover, because memory consolidation is believed to involve a gradual reorganization of the neural substrates underlying longterm memory storage (Bontempi et al., 1999), FGL could affect later structural processes regulating persistence of memory over time (Dudai, 2002). Both possibilities (affecting signaling transduction cascades or structural modifications, or both) seem to be plausible, as supported by the mechanisms of action described in vitro for FGL (see below).

Interestingly, we also observed an improved ability of FGLtreated rats to learn a new spatial learning response (reversal learning) at 2 weeks after training. At this time, they also displayed enhanced retrieval for the original platform location, indicating that the treatment did not prevent them from changing strategies and learning new processes. Hence the peptide seems to also facilitate behavioral flexibility and subsequent learning processes. This observation fits with the idea that better encoding of the environment leads to better retention and increased reactivity to a new spatial arrangement (Commins et al., 2003).

As a step toward the detailed dissection of the mechanisms underlying the memory-improving effects of FGL, our in vitro findings suggest that a facilitation of presynaptic function and an increase in the number of synapses might account for the behavioral effects of the peptide. Using hippocampal neuronal cultures, we show here that exposure to FGL, for either 1 or $48 \mathrm{hr}$, enhanced the rate of evoked FM1-43 unloading from presynaptic terminals in a dose-dependent manner. This suggests that FGL increases the probability of transmitter release from synaptic terminals in response to presynaptic depolarization. Such a mechanism is known to play a key role in short-term synaptic enhancement (Stevens and Wesseling, 1999). Furthermore, strong correlations between changes in quantal content and the magnitude of LTP have been described, during both early-phase (Liao et al., 1992) and late- phase (Sokolov et al., 2002) LTP. This supports the view that the mechanisms responsible for the expression and maintenance of synaptic potentiation include a presynaptic locus, i.e., enhanced transmitter release (Bliss and Collingridge, 1993).

A role for NCAM in the molecular organization and function of synaptic terminals has been suggested by studies of the neuromuscular junctions in NCAM-deficient mice (Polo-Parada et al., 2001; Ryan, 2001). Here, we also demonstrate that the facilitation of presynaptic function induced by the NCAM mimetic peptide, FGL, requires the activation of FGFR1. This finding is in agreement with in vitro evidence indicating that FGF2 can induce a rapid release of glutamate through the exocytotic system in a mitogen-activated protein kinase (MAPK) activation- dependent manner (Numakawa et al., 2002). Although FGL facilitated the release of neurotransmitter(s) that have not yet been identified, glutamatergic transmission is known to be predominant in synapses from primary cultures of hippocampal neurons (Li et al., 2002). In addition to facilitating synaptic function, evidence was also obtained suggesting that FGL induced the formation of new synapses in vitro, because the density of synaptophysin-positive spots was markedly increased by FGL treatment. This is consistent with the finding that treatment of cultures of hippocampal neurons with FGF2 increases the number of excitatory synapses by activating MAPK through FGFR (Li et al., 2002).

Although not tested in vivo, our in vitro findings suggest that the long-lasting facilitation of memory processes induced by FGL might be mediated by the ability of the peptide (via FGFR1 activation) to enhance presynaptic function or synapse formation, or both. Both mechanisms represent functional and structural forms of synaptic plasticity underlying long-term memory formation (Milner et al., 1998; Martin et al., 2000). Thus, posttraining FGL injections may exert their memory-enhancing effects either by assisting the synaptic changes triggered by the learning experience or by providing complementary substrates for the encoding of information, or both. It should be noted that such memory-improving effects appear to be highly specific for the ability of the FGL peptide to promote NCAM-FGFR interactions. Intracerebroventricular injections of $\mathrm{C} 3 \mathrm{~d}$, a peptide that binds to the IgI module of NCAM and disrupts NCAM-mediated cell adhesion (Kiryushko et al., 2003), induced the opposite effects in memory function, i.e., an impairment of memory consolidation for contextual fear conditioning (Cambon et al., 2003) and passive avoidance learning (Foley et al., 2000). In contrast to FGL, C3d has been shown to inhibit presynaptic function in vitro (Kiryushko et al., 2003).

The search for methods to improve cognitive abilities has interested humanity throughout history. Here, we provide strong evidence for cognition-enhancing properties of FGL in healthy adult rats. Future studies will address whether promoting NCAM interactions with FGFR1 might also facilitate memory processes and synaptic plasticity in cognitively impaired individuals.

\section{References}

Abel T, Lattal KM (2001) Molecular mechanisms of memory acquisition, consolidation and retrieval. Curr Opin Neurobiol 11:180-187.

Arami S, Jucker M, Schachner M, Welzl H (1996) The effect of continuous intraventricular infusion of L1 and NCAM antibodies on spatial learning in rats. Behav Brain Res 81:81-87.

Bliss TVP, Collingridge GL (1993) A synaptic model for memory: long-term potentiation in the hippocampus. Nature 361:31-39.

Bontempi B, Laurent-Demir C, Destrade C, Jaffard R (1999) Timedependent reorganization of brain circuitry underlying long-term memory storage. Nature 400:671-675.

Cambon K, Venero C, Berezin V, Bock E, Sandi C (2003) Post-training administration of a synthetic peptide ligand of the neural cell adhesion 
molecule, C3d, attenuates long-term expression of contextual fear conditioning. Neuroscience 122:183-191.

Commins S, Cunningham L, Harvey D, Walsh D (2003) Massed but not spaced training impairs spatial memory. Behav Brain Res 139:215-223.

Cremer H, Lange R, Christoph A, Plomann M, Vopper G, Roes J, Brown R, Baldwin S, Kraemer P, Scheff S, Barthels D, Rajewsky K, Wille W (1994) Inactivation of the NCAM gene in mice results in size reduction of the olfactory bulb and deficits in spatial learning. Nature 367:455-459.

Cremer H, Chazal G, Carleton A, Goridis C, Vincent JD, Lledo PM (1998) Long-term but not short-term plasticity at the mossy fiber synapses is impaired in neural cell adhesion molecule-deficient mice. Proc Natl Acad Sci USA 95:242-247.

Doyle E, Nolan PM, Bell R, Regan CM (1992) Intraventricular infusions of anti-neural cell adhesion molecules in discrete posttraining period impair consolidation of a passive avoidance response in the rat. J Neurochem 59:1570-1573.

Dudai Y (2002) Molecular bases of long-term memories: a question of persistence. Curr Opin Neurobiol 12:211-216.

Elgersma Y, Silva AJ (1999) Molecular mechanisms of synaptic plasticity and memory. Curr Opin Neurobiol 9:209-213.

Foley AG, Hartz BP, Gallagher HC, Rønn LC, Berezin V, Bock E, Regan CM (2000) A synthetic peptide ligand of neural cell adhesion molecule (NCAM) IgI domain prevents NCAM internalization and disrupts passive avoidance learning. J Neurochem 74:2607-2613.

Hiscock JJ, Murphy S, Willoughby JO (2000) Confocal microscopic estimation of GABAergic nerve terminals in the central nervous system. J Neurosci Methods 95:1-11.

Kim JJ, Fanselow M (1992) Modality-specific retrograde amnesia of fear. Science 256:675-676.

Kiryushko D, Kofoed T, Skladchikova G, Holm A, Berezin V, Bock E (2003) A synthetic peptide ligand of NCAM, C3d, promotes neuritogenesis and synaptogenesis and modulates presynaptic function in primary cultures of rat hippocampal neurons. J Biol Chem 278:12325-12334.

Kiselyov V, Skladchikova G, Hinsby AM, Jensen PH, Kulahin N, Soroka V, Pedersen N, Tsetlin V, Poulsen FM, Berezin V, Bock E (2003) Structural basis for a direct interaction between FGFR 1 and NCAM and evidence for a regulatory role of ATP. Structure 11:691-701.

Kolkova K, Novitskaya V, Pedersen N, Berezin V, Bock E (2000) Neural cell adhesion molecule-stimulated neurite outgrowth depends on activation of protein kinase $\mathrm{C}$ and the Ras-mitogen-activated protein kinase pathway. J Neurosci 20:2238-2246.

Li AJ, Suzuki S, Suzuki M, Mizukoshi E, Imamura T (2002) Fibroblast growth factor- 2 increases functional excitatory synapses on hippocampal neurons. Eur J Neurosci 16:1313-1324.

Liao D, Jones A, Malinow R (1992) Direct measurement of quantal changes underlying long-term potentiation in CA1 hippocampus. Neuron 9:1089-1097.

Luthi A, Laurent JP, Figurov A, Muller D, Schachner M (1994) Hippocampal long term potentiation and neural cell adhesion molecules L1 and NCAM. Nature 372:777-779.

Maar TE, Rønn LCB, Bock E, Berezin V, Moran J, Pasantes-Morales H, Schousboe A (1997) Characterization of microwell cultures of dissociated brain tissue for studies of cell-cell interactions. J Neurosci Res 47:163-172.

Malenka RC, Nicoll RA (1999) Long-term potentiation: a decade of progress? Science 285:1870-1874.

Martin SJ, Morris RG (2002) New life in an old idea: the synaptic plasticity and memory hypothesis revisited. Hippocampus 12:609-636.

Martin SJ, Grimwood PD, Morris RG (2000) Synaptic plasticity and memory: an evaluation of the hypothesis. Annu Rev Neurosci 23:649-711.

Merino JJ, Cordero MI, Sandi C (2000) Regulation of hippocampal cell adhesion molecules NCAM and L1 by contextual fear conditioning is dependent upon time and stressor intensity. Eur J Neurosci 12:3283-3290.

Mileusnic R, Rose SPR, Lancashire C, Bullock S (1995) Characterisation of antibodies specific for chick brain neural cell adhesion molecules which cause amnesia for a passive avoidance task. J Neurochem 64:2598-2606.

Milner B, Squire LR, Kandel ER (1998) Cognitive neuroscience and the study of memory. Neuron 20:445-468.

Morris RG, Garrud P, Rawlins JN, O'Keefe J (1982) Place navigation impaired in rats with hippocampal lesions. Nature 297:681-683.

Mosevitsky MI, Capony JP, Skladchikova GY, Novitskaya VA, Plekhanov AY, Zakharov VV (1997) The BASP1 family of myristoylated proteins abundant in axonal termini. Primary structure analysis and physico-chemical properties. Biochimie 79:373-384.
Niethammer P, Delling M, Sytnyk V, Dityatev A, Fukami K, Schachner M (2002) Cosignaling of NCAM via lipid rafts and the FGF receptor is required for neuritogenesis. J Cell Biol 157:521-532.

Numakawa T, Yokomaku D, Kiyosue K, Adachi N, Matsumoto T, Numakawa Y, Taguchi T, Hatanaka H, Yamada M (2002) Basic fibroblast growth factor evokes a rapid glutamate release through activation of the MAPK pathway in cultured cortical neurons. J Biol Chem 277:28861-28869.

Phillips RG, Ledoux JE (1992) Differential contribution of amygdala and hippocampus to cued and contextual fear conditioning. Behav Neurosci 106:274-285.

Polo-Parada L, Bose CM, Landmesser LT (2001) Alterations in transmission, vesicle dynamics, and transmitter release machinery at NCAMdeficient neuromuscular junctions. Neuron 31:815-828.

Quirk GJ, Armony JL, LeDoux JE (1997) Fear conditioning enhances different temporal components of the tone-evoked spike trains in auditory cortex and lateral amygdala. Neuron 19:613-624.

Rescorla RA (1996) Preservation of Pavlovian association through extinction. Q J Exp Psychol 49B:245-258.

Rønn LCB, Bock E, Linnemann D, Jahnsen H (1995) NCAM-antibodies modulate induction of long-term potentiation in rat hippocampal CA1. Brain Res 677:145-151.

Rønn LCB, Olsen M, Ostergaard S, Kiselyov V, Berezin V, Mortensen MT, Lerche MH, Jensen PH, Soroka V, Saffell JL, Doherty P, Poulsen FM, Bock E, Holm A (1999) Identification of a neuritogenic ligand of the neural cell adhesion molecule using a combinatorial library of synthetic peptides. Nature Biotech 17:1000-1005.

Rønn LCB, Berezin V, Bock E (2000a) The neural cell adhesion molecule in synaptic plasticity and ageing. Int J Dev Neurosci 18:193-199.

Rønn LCB, Ralets I, Hartz B, Beck M, Berezin A, Berezin V, Møller A, Bock E (2000b) A simple procedure for quantification of neurite outgrowth based on stereological principles. J Neurosci Methods 100:25-32.

Rønn LCB, Dissing S, Holm A, Berezin V, Bock E (2002) Increased intracellular calcium is required for neurite outgrowth induced by a synthetic peptide ligand of NCAM. FEBS Lett 518:60-66.

Roozendaal B, McGaugh JL (1997) Basolateral amygdala lesions block the memory-enhancing effect of glucocorticoid administration in the dorsal hippocampus of rats. Eur J Neurosci 9:76-83.

Ryan TA (2001) NCAM and vesicle cycling: the importance of good glue in the long run. Neuron 32:759-761.

Ryan TA, Reuter H, Wendland B, Schweizer FE, Tsien RW, Smith SJ (1993) The kinetics of synaptic vesicle recycling measured at single presynaptic boutons. Neuron 11:713-724.

Sacchetti B, Baldi E, Ambrogi L, Bucherelli C (2002) Differential contribution of some cortical sites to the formation of memory traces supporting fear conditioning. Exp Brain Res 146:223-232.

Saffell JL, Walsh FS, Doherty P (1994) Expression of NCAM containing VASE in neurons can account for a developmental loss in their neurite outgrowth response to NCAM in a cellular substratum. J Cell Biol 125:427-436.

Sandi C (1998) Role and mechanisms of action of glucocorticoids in memory formation. Neural Plast 6:39-49.

Sandi C, Rose SPR, Mileusnic R, Lancashire C (1995) Corticosterone facilitates long-term memory formation via enhanced glycoprotein synthesis. Neuroscience 69:1087-1093.

Schachner M (1997) Neural recognition molecules and synaptic plasticity. Curr Opin Cell Biol 9:627-634.

Sokolov MV, Rossokhin AV, Astrelin AV, Frey JU, Voronin LL (2002) Quantal analysis suggests strong involvement of presynaptic mechanisms during the initial $3 \mathrm{~h}$ maintenance of long-term potentiation in rat hippocampal CA1 area in vitro. Brain Res 957:61-75.

Soroka V, Kiryushko D, Novitskaya V, Rønn LCB, Poulsen FM, Holm A, Bock E, Berezin V (2002) Induction of neuronal differentiation by a peptide corresponding to the homophilic binding site of the second Ig module of the neural cell adhesion molecule. J Biol Chem 277:24676-24683.

Stevens CF, Wesseling JG (1999) Augmentation is a potentiation of the exocytotic process. Neuron 22:139-146.

Torras-Garcia M, Portell-Cortes I, Costa-Miserachs D, Morgado Bernal I (1997) Long-term memory modulation by post-training epinephrine in rats: differential effects depending on the basic learning capacity. Behav Neurosci 111:301-308.

Zakharenko SS, Zablow L, Siegelbaum SA (2001) Visualization of changes in presynaptic function during long-term synaptic plasticity. Nat Neurosci 4:711-717. 